\title{
Enhanced protopanaxadiol production from xylose by engineered Yarrowia lipolytica
}

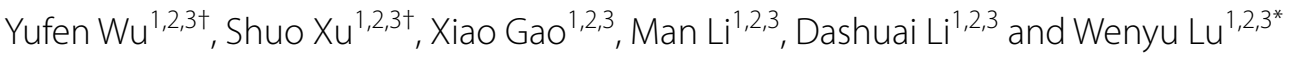

\begin{abstract}
Background: As renewable biomass, lignocellulose remains one of the major choices for most countries in tackling global energy shortage and environment pollution. Efficient utilization of xylose, an important monosaccharide in lignocellulose, is essential for the production of high-value compounds, such as ethanol, lipids, and isoprenoids. Protopanaxadiol (PPD), a kind of isoprenoids, has important medical values and great market potential.

Results: The engineered protopanaxadiol-producing Yarrowia lipolytica strain, which can use xylose as the sole carbon source, was constructed by introducing xylose reductase (XR) and xylitol dehydrogenase (XDH) from Scheffersomyces stipitis, overexpressing endogenous xylulose kinase (yIXKS) and heterologous PPD synthetic modules, and then $18.18 \mathrm{mg} / \mathrm{L}$ of PPD was obtained. Metabolic engineering strategies such as regulating cofactor balance, enhancing precursor flux, and improving xylose metabolism rate via XR (K270R/N272D) mutation, the overexpression of tHMG1/ERG9/ERG20 and transaldolase (TAL)/transketolase (TKL)/xylose transporter (TX), were implemented to enhance PPD production. The final Y14 strain exhibited the greatest PPD titer from xylose by fed-batch fermentation in a 5-L fermenter, reaching $300.63 \mathrm{mg} / \mathrm{L}$ [yield, $2.505 \mathrm{mg} / \mathrm{g}$ (sugar); productivity, $2.505 \mathrm{mg} / \mathrm{L} / \mathrm{h}$ ], which was significantly higher than the titer of glucose fermentation [titer, $167.17 \mathrm{mg} / \mathrm{L}$; yield, $1.194 \mathrm{mg} / \mathrm{g}$ (sugar); productivity, $1.548 \mathrm{mg} / \mathrm{L} / \mathrm{h}]$.

Conclusion: The results showed that xylose was more suitable for PPD synthesis than glucose due to the enhanced carbon flux towards acetyl-CoA, the precursor for PPD biosynthetic pathway. This is the first report to produce PPD in Y. lipolytica with xylose as the sole carbon source, which developed a promising strategy for the efficient production of high-value triterpenoid compounds.
\end{abstract}

Keywords: Protopanaxadiol, Xylose, Yarrowia lipolytica, Metabolic engineering, Synthetic biology

\section{Background}

Lignocellulose from wood in forestry and agriculture as well as industrial waste can reach 100 billion tons/ year, making this biomass the most abundant renewable resource on the Earth [1]. Efficient utilization of lignocellulose is essential for reducing demands for energy and food. Xylose is the second most abundant monosaccharide in lignocellulosic hydrolysate following glucose, accounting for nearly $35 \%$ of all monosaccharides [2]. However, most microorganisms cannot efficiently

\footnotetext{
*Correspondence: wenyulu@tju.edu.cn

'Yufen Wu and Shuo Xu contributed equally to this work

${ }^{1}$ School of Chemical Engineering and Technology, Tianjin University,

Tianjin, People's Republic of China

Full list of author information is available at the end of the article
}

metabolize xylose from lignocellulosic hydrolysate due to the carbon catabolite repression effect [3], which largely limits the applications of lignocellulose. Thus, the use of xylose has become a hot issue in the study of lignocellulose.

In recent years, great progress has been made in xylose metabolism studies. Various compounds have been successfully obtained via microbial metabolism of xylose, such as xylitol [4], ethanol [5], acetoin [6], fumaric acid [7], and polyhydroxyalkanoate [8]. As a model organism for lipid metabolism, Yarrowia lipolytica does not naturally metabolize xylose, primarily due to low expression levels of the key enzymes involved in the xylose metabolic pathway [9-12]. However, whether the Y. lipolytica strain can grow using xylose as a substrate remains controversial [13-15]. There have been many attempts to engineer 
Y. lipolytica to use xylose as a substrate. Ledesma-Amaro et al. [16] have engineered Y. lipolytica to metabolize xylose to produce lipids and citric acid by overexpression of xylose reductase (XR) and xylitol dehydrogenase $(\mathrm{XDH})$ from Scheffersomyces stipitis and endogenous xylulose kinase (ylXKS). The growth ability of engineered $Y$. lipolytica using xylose was identical to that of the wild type strain grown using glucose. This mutant could produce up to $80 \mathrm{~g} / \mathrm{L}$ of citric acid from xylose. Li and Alper [17] have implemented a starvation adaptation strategy to improve the metabolic rate of xylose. They introduced a heterologous oxidoreductase pathway to optimize xylose utilization by Y. lipolytica in a stable manner; this mutant produced $>15 \mathrm{~g} / \mathrm{L}$ of lipid via bioreactor fermentation, with a maximal lipid productivity of $0.19 \mathrm{~g} / \mathrm{L} / \mathrm{h}$.

Yarrowia lipolytica contains the native mevalonate (MVA) pathway to provide precursor compounds, namely isopentenyl pyrophosphate and dimethylallyl pyrophosphate [18], indicating that $Y$. lipolytica can serve as a natural host for terpenoid synthesis. Y. lipolytica is a potential platform for producing isoprenoids using acetyl-CoA as the precursor due to its convenient genetic manipulation, robust acetyl-CoA synthesis, NADPH, and energy supply system [19-22], as $S$. cerevisiae lacks acetyl-CoA [23, 24]. In previous studies, biosynthesis of many terpenoids were realized in $Y$. lipolytica, such as farnesene, limonene, and ginsenoside compound $\mathrm{K}$ [25-27], and engineering strategies have been employed to enhance the production, such as codon optimization, heterologous synthetic genes introduction, synthetic pathway up-regulation, and competitive pathway downregulation. Limonene $(23.56 \mathrm{mg} / \mathrm{L} ; 1.36 \mathrm{mg} / \mathrm{g} \mathrm{DCW})$ was obtained in $Y$. lipolytica by codon optimization and overexpression HMG1 and ERG12 genes [25]; The $\alpha$-farnesene titer was increased by 20.8 -fold by $t H M G 1$, $I D I$, and ERG20 overexpression, reaching $259.98 \mathrm{mg} / \mathrm{L}$ [26]; The titer of ginsenoside compound $\mathrm{K}$ was increased to $161.8 \mathrm{mg} / \mathrm{L}$ by a combination of metabolic engineering strategies [27].

Protopanaxadiol (PPD) is a natural $\mathrm{C}_{30}$ isoprenoid with important medical applications owing to its anticancer, antitumor, antiviral, and antibiotic properties [28-30]. The extraction method for PPD from plants has limited applications because of shortage of ginseng plants. Therefore, strategies have been developed for PPD biosynthesis to overcome the limitations of traditional extraction processes [31, 32].

Xylose fermentation is more desirable for isoprenoid production than glucose fermentation by Saccharomyces cerevisiae due to a rigid flux partition toward ethanol during glucose metabolism [33-35]. However, there are challenges that must be overcome during isoprenoid production from xylose in S. cerevisiae, such as cofactor imbalance, slow xylose metabolism rate, and insufficient acetyl-CoA supply. In this study, Y. lipolytica was selected as the host for PPD production from xylose due to robust acetyl-CoA synthesis, NADPH, and energy supply system. Engineered $Y$. lipolytica strains that could metabolize xylose were constructed to evaluate the metabolic capacity of xylose by introducing XR and XDH from S. stipitis or from $Y$. lipolytica. PPD production was enhanced by overexpressing specific components of the MVA pathway, enhancing the xylose transport rate, and strengthening the metabolic pathway (Fig. 1). Fermentation was performed using xylose, glucose, and mixed sugar as carbon sources to evaluate the performance of different substrates to produce PPD titers. Finally, fermentation amplification was conducted in a 5 - $\mathrm{L}$ bioreactor to examine the stability xylose metabolism for PPD production.

\section{Results and discussion}

Ku70 knockout to enhance homologous recombination efficiency of $Y$. lipolytica

Yarrowia lipolytica is a promising industrial producer of lipids due to its fully annotated genome, ease of manipulation, and ability to utilize hydrophobic carbon sources as substrates [36, 37]. However, homologous integration of exogenous DNA can be difficult because $Y$. lipolytica mainly prefers the non-homologous end-joining (NHEJ) recombination rather than the homologous recombination (HR) [38]. Lustig [39] has confirmed that both KU70 and KU80 bind to broken DNA ends exhibit bridging activity regardless of the sequence homology of the broken ends. To increase the rate of HR in Y. lipolytica, Ku70 deletion cassettes with the LoxP-URA3-LoxP marker were generated to block the NHEJ pathway and were transformed into Y. lipolytica ATCC 201249 to obtain the Y1 strain. HR efficiency increased from 28 to 54\% after Ku70 knockout. Shake flask fermentation (Fig. 2a) revealed no difference in cell growth, indicating that Ku70 knockout had a great effect in improving the HR efficiency of $Y$. lipolytica and did not adversely affect cell growth, which is important for the construction of engineered strains. In previous studies, the HR frequency is also dramatically increased in Y. lipolytica by disrupting the ku70 gene [40, 41]. However, Ku70 knockout strain may have higher instability than wild type. The alternative approaches to transiently downregulate $k u 70$ can be considered to improve gene integration, which have been confirmed in Trichoderma reesei [42].

\section{Introduction of the xylose metabolic pathway in $Y$. lipolytica}

The natural capacity of $Y$. lipolytica to metabolize xylose remains unclear. To obtain xylose-fermenting strains, 


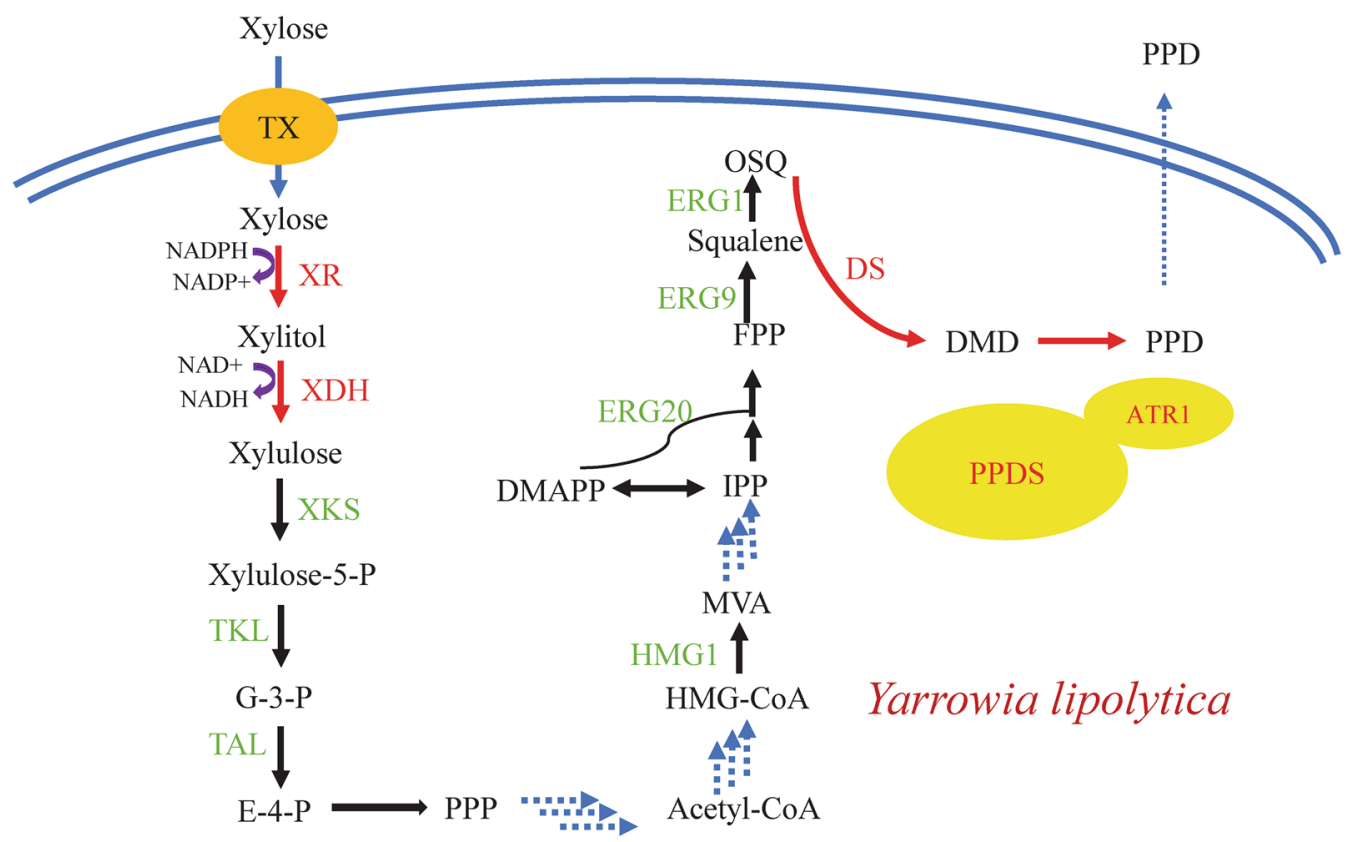

Fig. 1 Metabolic engineering strategies for producing PPD from xylose. TX, sugar transporter; XR, xylose reductase; XDH, xylitol dehydrogenase; XKS, Xylulose kinase; TKL, transketolase; TAL, transaldolase; HMG1, 3-hydroxy-3-methylglutaryl-coenzyme A reductase; ERG9, squalene synthase; ERG20, farnesyl pyrophosphate synthase; ERG1, squalene monooxygenase; DS, DMD synthase; PPDS, PPD synthase; ATR1, NADPH-P450 reductase; G-3-P, glyceraldehyde-3-phosphate; E-4-P, erythrose-4-phosphate; PPP, pentose phosphate pathway; HMG-CoA, hydroxymethylglutaryl-CoA; MVA, mevalonate acid; OSQ, 2,3-oxidized squalene; DMD, dammarenediol-II; PPD, protopanaxadiol. Green represents the overexpressed native genes and red represents the optimized heterologous genes. Three dotted arrows represent multi-step reactions

endogenous and heterologous pathways for xylose metabolism were introduced into $Y$. lipolytica. The cofactor imbalance problem can be resolved using mutated XR [43-45]. Watanabe et al. [43] have induced two XR gene mutations, $\mathrm{R} 276 \mathrm{H}$ and $\mathrm{K} 270 \mathrm{R} / \mathrm{N} 272 \mathrm{D}$, resulting in change in preference from NADPH to NADH, which led to respectively 52 - and 146-fold increases in catalytic efficiency of the mutant strains compared with that of the original strain. $X Y L 1$ and $X Y L 2$ genes from S. stipitis encoding XR (K270R/N272D) and XDH, respectively, were codon-optimized according to codon preference of $Y$. lipolytica and transformed into the Y1 strain, together with the $y l X K S$ expression cassette, to produce the strain Y2 (Fig. 1). Moreover, the endogenous $y l X Y L 1, y l X Y L 2$, and $y l X K S$ genes were integrated into the zeta site of $Y$. lipolytica using the same promoters as those of the heterologous genes to obtain the Y3 strain. Y. lipolytica ATCC 201249 could not grow in xylose medium (Fig. 2a). The Y2 series of engineered strains with the introduced heterologous xylose metabolic pathway showed a significant increase in $\mathrm{OD}_{600}$ values compared with the original strain, indicating that XR and XDH derived from S. stipitis were successfully expressed in Y. lipolytica. However, the Y3 strain overexpressing the endogenous xylose metabolism genes showed no obvious growth, indicating that endogenous $\mathrm{XR}$ and $\mathrm{XDH}$ cannot use xylose as the sole carbon source.

Strain with the highest $\mathrm{OD}_{600}$ using xylose was selected for shake flask fermentation, with $\mathrm{Y} 0$ and $\mathrm{Y} 1$ as comparisons. Results indicated that the Y2 strain alone consumed approximately $10 \mathrm{~g} / \mathrm{L}$ xylose and that the growth ability using xylose remained lower than that using glucose (Fig. 2b, c). Therefore, we speculated that improving the efficiency of sugar transporters [46-48] or enhancing the adaptability of strains to xylose [49] can enhance xylose utilization.

\section{Adaptation to improve xylose metabolism}

Studies have reported that $Y$. lipolytica, with introduced heterologous xylose metabolic pathway, can utilize xylose, although the phenotype of xylose catabolism is unstable [50]. Therefore, we used the adaption strategy to overcome the instability of $Y$. lipolytica growth in xylose. The strains $\mathrm{Y} 2$ and $\mathrm{Y} 3$ were cultured repeatedly in SC medium containing xylose for a long time, and obtained strains Y4 and Y5, respectively. The Y5 strain showed no significant increase in $\mathrm{OD}_{600}$ compared with the $\mathrm{Y} 1$ strain, whereas the $\mathrm{OD}_{600}$ of the $\mathrm{Y} 4$ strain reached 20.12 (Fig. 3a). In addition, Y4 consumed $20 \mathrm{~g} / \mathrm{L}$ xylose in $72 \mathrm{~h}$, and the rate of xylose metabolism increased 1.69-folds 

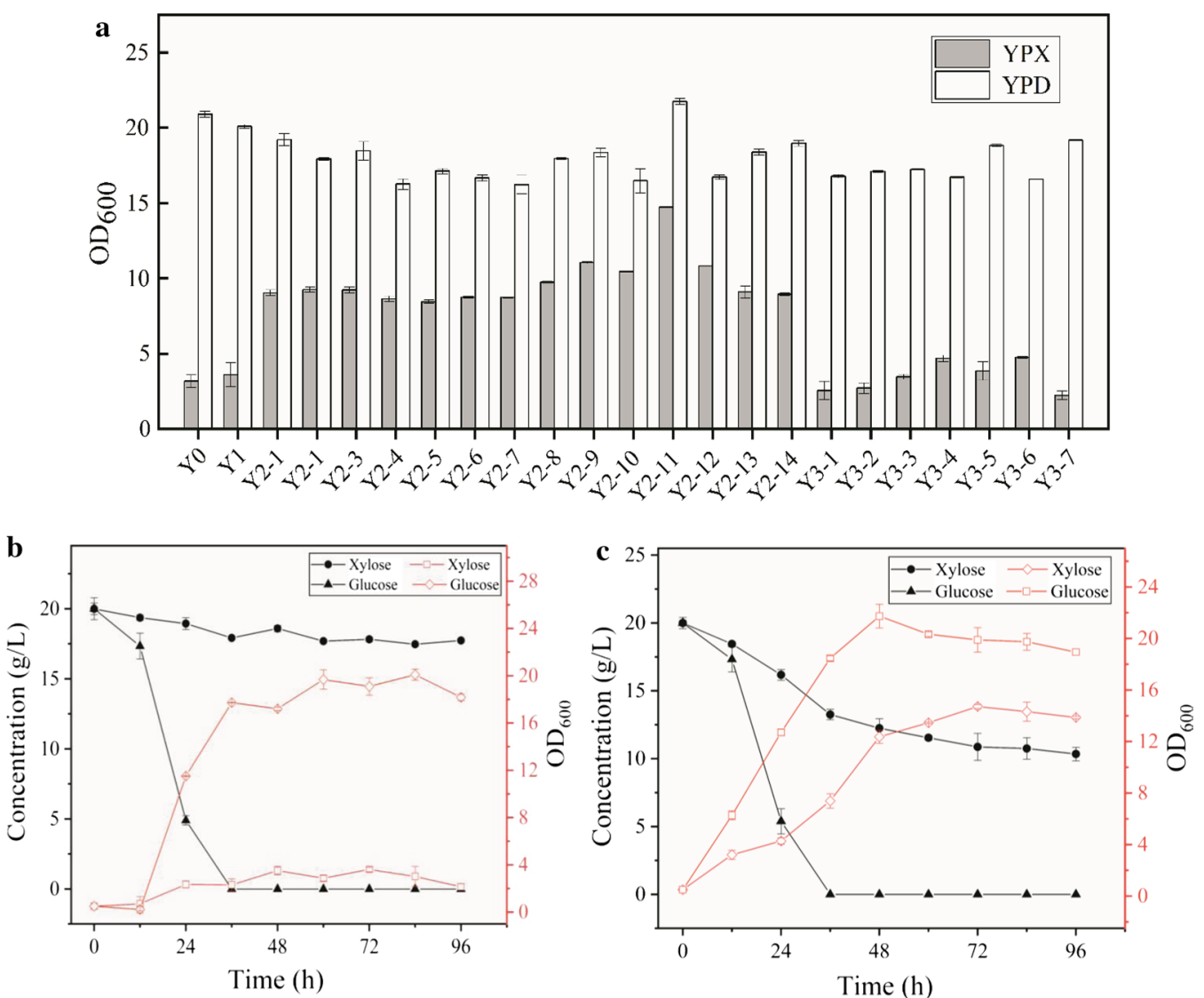

Fig. 2 The growth of different engineered strains from xylose and glucose, respectively. a The $\mathrm{OD}_{600}$ of Y0 (Yarrowia lipolytica ATCC 201249), Y1 (Ku70 deletion), Y2-1_Y2-14 (introducing heterologous xylose metabolic pathway) and Y3-1_Y3-7 (introducing endogenous metabolic pathway) in YPD and YPX medium, respectively. $\mathbf{b}$ The $\mathrm{OD}_{600}$ and sugar concentration curves of Y1 from xylose and glucose as substrates, respectively. $\mathbf{c}$ The $\mathrm{OD}_{600}$ and sugar concentration curves of $\mathrm{Y} 2$ from xylose and glucose as substrates, respectively. Each data represents an average \pm 1 standard deviation of three parallel fermentations
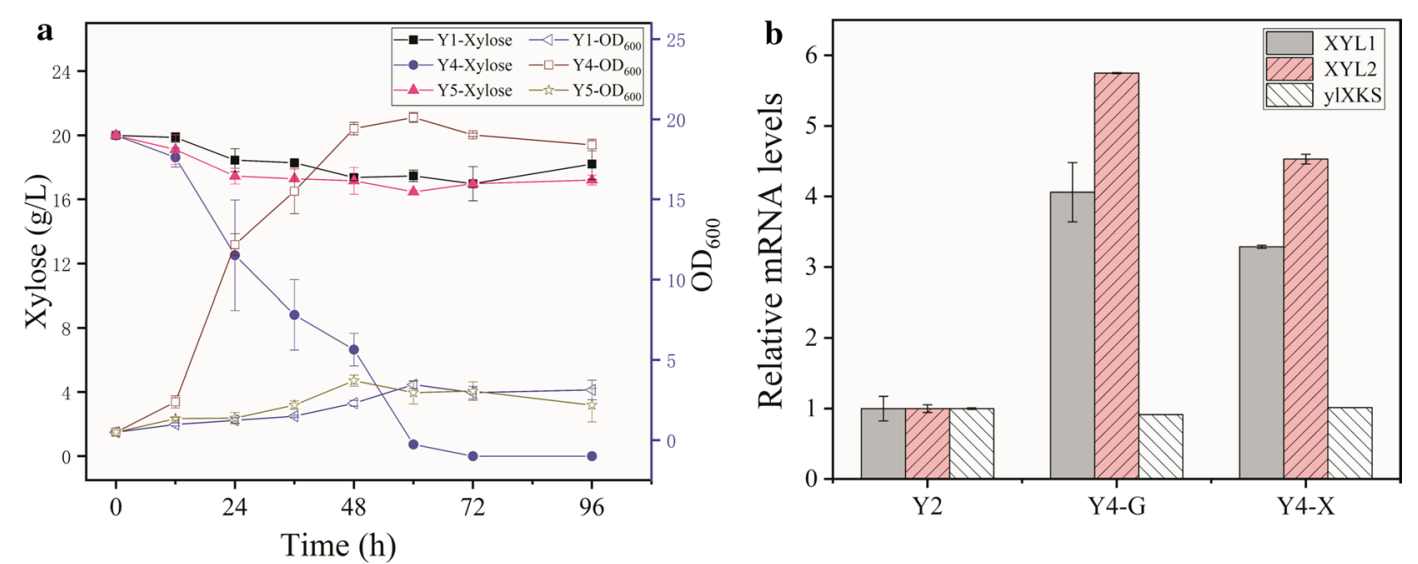

Fig. 3 The effects of different strains after adaptation. a The $\mathrm{OD}_{600}$ and sugar concentration curves of $Y 4$ and $Y 5$ by adaptation of $Y 2$ and $Y 3$, respectively, compared with Y1. b Relative mRNA expression levels of $X Y L 1, X Y L 2$ and $y \mid X K S$ at exponential phase for strain $Y 2$ and $Y 4$, were measured with RT-qPCR. Y2 were grown on glucose. Y4 was grown on either glucose and xylose. The relative mRNA levels of the strain $Y 2$ were 1. Each data represents an average \pm 1 standard deviation of three parallel fermentations 
the rate by Y2. RT-qPCR was performed to analyze the difference in the rate of xylose metabolism after adaptation. We observed that the expression levels of exogenous $X Y L 1$ and XYL2 in Y4 were indeed much higher than the strain Y2 (Fig. 3b), which would be beneficial for promoting xylose metabolism. We also measured the expression level of $y l X K S$ under the same conditions, and observed no substantial changes of $y l X K S$ expression between the strains (Fig. 3b). These results indicated that adaptation to xylose could significantly promote xylose utilization and improve the stability of Y. lipolytica in xylose, which was probably due to the improvement of XYL1 and XYL2 expression levels after adaptation.

\section{PPD production in xylose-metabolizing strains of $Y$. lipolytica}

The leu 2 and ura 3 markers were used in the construction of engineered strain Y4. To recycle the marker gene ura3, the CRE-expressing plasmid pINA1269-CRE was used, resulting in the Y6 strain. The PPD synthesis pathway including dammarenediol-II (DS) synthase, cytochrome P450 monooxygenase (PPDS), and NADPH-P450 reductase (ATR1), has previously been successfully expressed in Y. lipolytica [27]. In this study, co-expression and fusion expression modules (Additional file 1: Figure S1) for PPD synthesis were integrated into the $Y$. lipolytica $\mathrm{Y} 6$ genome, as previously described, resulting in the $\mathrm{Y} 7$ and $\mathrm{Y} 8$ strains, respectively. The $\mathrm{Y} 7$ and $\mathrm{Y} 8$ strains performed fermentation in YPX medium while shaking (Fig. 4a, b). In YPX medium, PPD titer from strain Y8 was $60.1 \mathrm{mg} / \mathrm{L}$, and increased 2.3 times compared with Y7 (18.18 mg/L). PPD yield from Y8 by fusion expression was significantly higher than that from $\mathrm{Y} 7$ by co-expression, indicating that the difference in PPD yield between the two strains was due to expression of the PPDS and ATR1. Increasing the precursor supply may further increase PPD production.

\section{Overexpression of the MVA pathway to enhance PPD production}

The MVA pathway is the only source of precursors for the synthesis of terpenoids in Y. lipolytica [51-53]. Several key enzymes in the MVA pathway have been investigated to increase PPD production in S. cerevisiae or
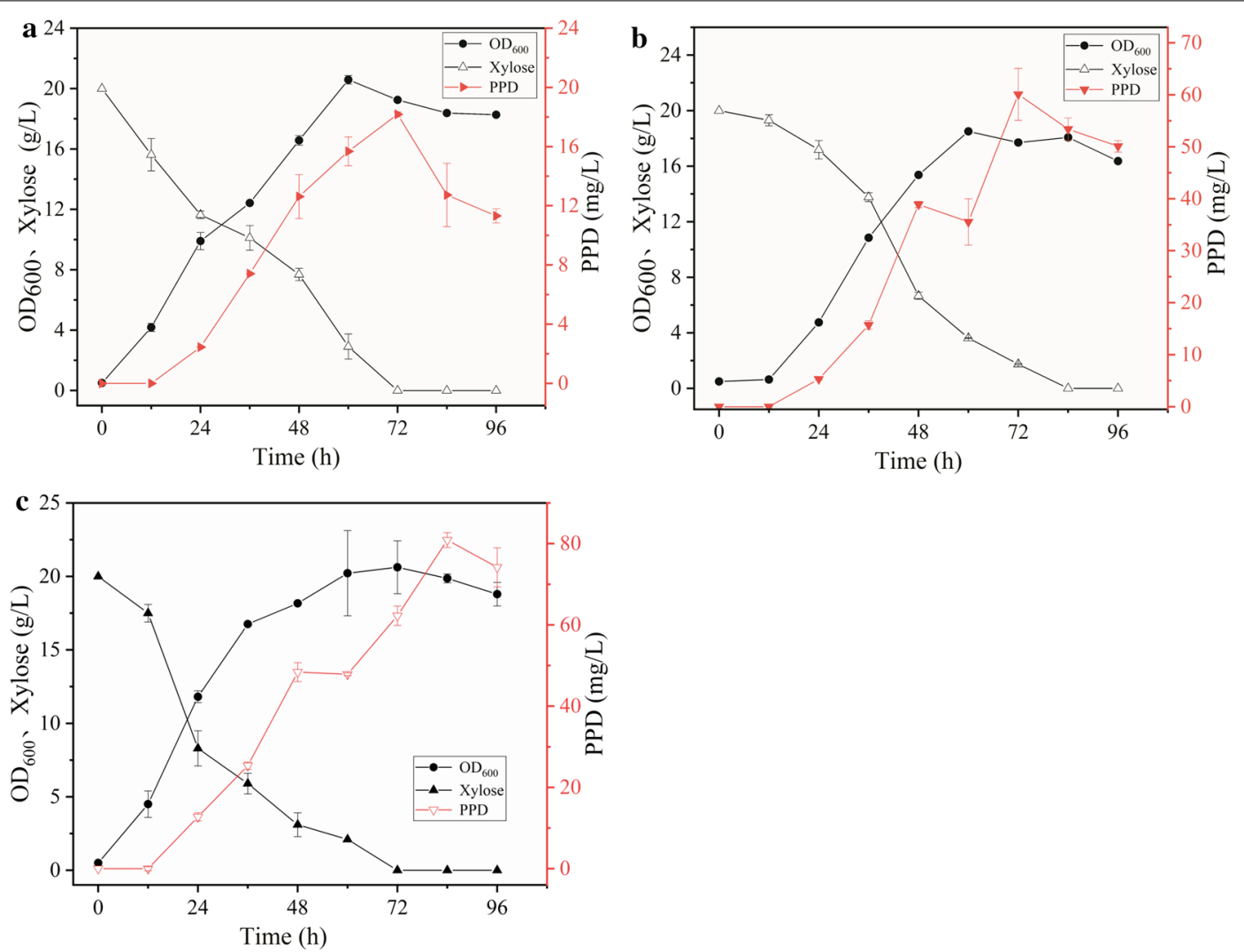

Fig. 4 The production of PPD in different strains utilizing xylose. a The production of PPD in strain Y7 by co-expression of PPD synthesis modules. b The production of PPD in strain Y8 by fusion expression PPD synthesis modules. $\mathbf{c}$ Fermentation of strain $\mathrm{Y} 10$ overexpressing MVA pathway for enhancing PPD production at shake flask level. Each data represents an average \pm 1 standard deviation of three parallel fermentations 
Y. lipolytica, such as tHMG1, ERG10, ERG12, ERG9, ERG20, and ERG13 [32, 35]. The key enzymes, tHMG1, ERG9, and ERG20, were overexpressed in Y9 (the ura3 marker in Y8 was recycled), resulting in the Y10 strain, in which PPD titer increased by $34 \%$ compared with that in the Y8 strain, reaching $80.88 \mathrm{mg} / \mathrm{L}$, although $\mathrm{OD}_{600}$ of the cells did not change significantly (Fig. 4c). Overexpression of the MVA pathway significantly increased PPD production; however, in Y. lipolytica, PPD yield from xylose could be further increased by increasing the metabolic rate of xylose.

\section{Optimization of the xylose assimilation pathway to increase the xylose metabolism rate}

Optimization of the xylose assimilation pathway involves overexpression of xylose transporters to increase the xylose transport rate and improve the flux of xylulose into the pentose phosphate pathway. The xylose transporter in Y. lipolytica is in a dormant state; however, the xylose assimilation rate was enhanced by approximately $50 \%$ by overexpressing xylose transporters (YALI0B00396p) [54]. Meanwhile, acetyl-CoA is a basic precursor for terpenoid synthesis. Xylose needs to enter the central carbon metabolism via the pentose phosphate pathway, through which acetyl-CoA is derived. Transaldolase (TAL) and transketolase (TKL) - the key enzymes entering the pentose phosphate pathway-are immediately downstream of the xylose degradation pathway. TAL and TKL have been successfully expressed in $S$. cerevisiae to promote xylose metabolism $[35,55]$. In the present study, the ura3 marker in the Y10 strain was recycled to obtain the Y11 strain. Endogenous TAL and TKL genes were overexpressed in the Y11 strain via HR, resulting in the Y12 strain. The ura3 marker in Y12 was recycled to obtain the Y13 strain. The Y14 strain was obtained from the
Y13 strain by introducing TX (YALI0B00396p) expression modules. Fermentation results are summarized in Fig. 5. $\mathrm{OD}_{600}$ of the Y12 strain increased to 25.03 , indicating that the biomass improved as a result of overexpression of $T A L$ and $T K L$. In addition, PPD titer increased to $88.73 \mathrm{mg} / \mathrm{L}$, indicating that the flux of xylose to the central pentose phosphate pathway had increased. The overexpression of xylose transporter (TX) decreased PPD yield (about $80 \mathrm{mg} / \mathrm{L}$ ), however, $\mathrm{OD}_{600}$ increased significantly (32.81), indicating that the metabolic rate of xylose had increased and that more carbon source was converted to biomass.

\section{Shake flask fermentation of the engineered strain Y14 using xylose to produce PPD}

Mixed sugar fermentation is often used as an effective strategy to improve the comprehensive utilization of sugars $[50,56,57]$. To explore the feasibility of mixed sugar fermentation by $Y$. lipolytica, optimization of the initial sugar concentration and fermentation of mixed sugar were performed using the engineered Y14 strain. The Y14 strain was allowed to ferment initial sugar concentrations of $20,30,40,50,60,70$, and $80 \mathrm{~g} / \mathrm{L}$, respectively, in YPD and YPX media for 4 days. $\mathrm{OD}_{600}$ at a concentration of $60 \mathrm{~g} / \mathrm{L}$ xylose was the maximum, reaching 34.3 (Table 1). The initial concentration of xylose consumed by the Y14 strain was $40 \mathrm{~g} / \mathrm{L}$, and PPD yield using $40 \mathrm{~g} / \mathrm{L}$ xylose was significantly higher than that using low sugar concentrations. In addition, at different sugar concentrations, PPD titer using xylose was higher than that using glucose, indicating that the biomass and PPD production by the Y14 strain using xylose were better than those using glucose. In terms of biomass and PPD production, $40 \mathrm{~g} / \mathrm{L}$ was selected as the initial optimal sugar concentration for fermentation of xylose and glucose by the Y14 strain.
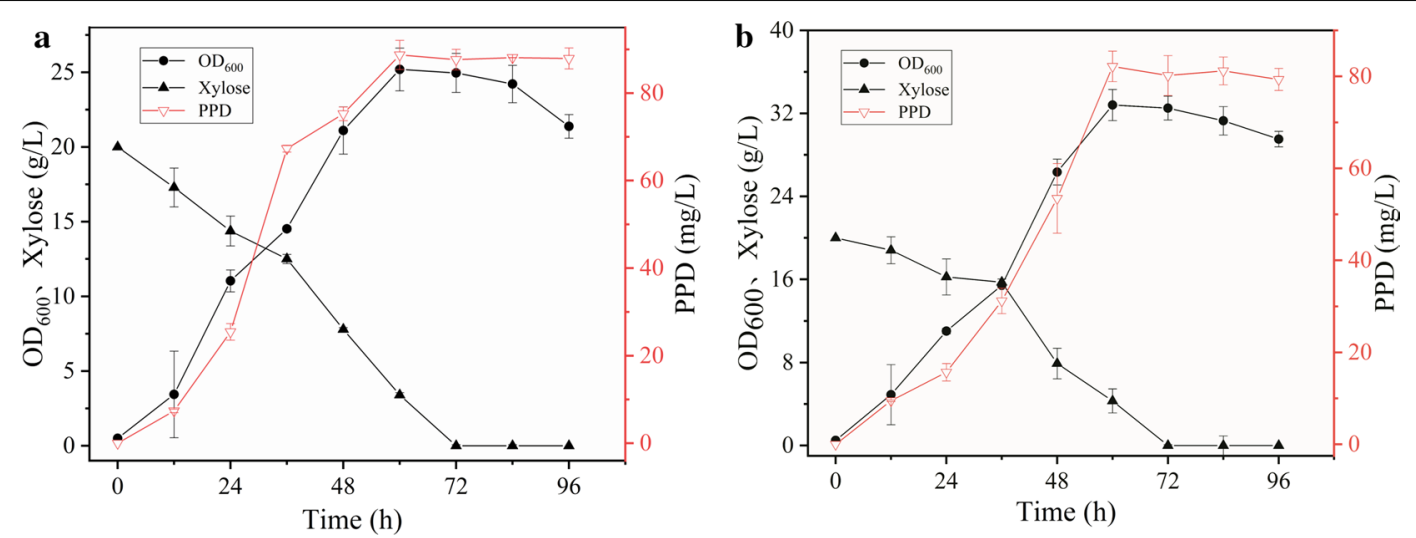

Fig. 5 Shake flask fermentation of engineered strains that enhanced the xylose metabolic pathway for PPD production. a Fermentation of strains Y12 overexpressing TKL and TAL for improving PPD production from xylose. b Fermentation of strains Y14 overexpressing TX for improving PPD production from xylose. Each data represents an average \pm 1 standard deviation of three parallel fermentations 
Table 1 Fermentation of Y14 at different initial sugar concentrations

\begin{tabular}{lllll}
\hline $\begin{array}{l}\text { Initial sugar } \\
\text { concentration } \\
\text { (g/L) }\end{array}$ & Symbol $^{\mathbf{a}}$ & $\mathbf{O D}_{\mathbf{6 0 0}}$ & $\begin{array}{l}\text { Residual sugar } \\
\text { concentration } \\
(\mathbf{g} / \mathbf{L})^{\mathbf{b}}\end{array}$ & $\begin{array}{l}\mathbf{P P D} \text { titer } \\
(\mathbf{m g} / \mathbf{L})^{\mathbf{b}}\end{array}$ \\
\hline 20 & $\mathrm{G}$ & 21.7 & 0 & $57.17 \pm 1.83$ \\
& $\mathrm{X}$ & 26.8 & 0 & $81.23 \pm 1.47$ \\
30 & $\mathrm{G}$ & 29.6 & 0 & $61.17 \pm 1.31$ \\
& $\mathrm{X}$ & 24.1 & 0 & $80.45 \pm 0.71$ \\
40 & $\mathrm{G}$ & 27.4 & 0 & $65.27 \pm 1.28$ \\
& $\mathrm{X}$ & 30.5 & 0 & $120.36 \pm 1.59$ \\
50 & $\mathrm{G}$ & 30.3 & 0 & $58.62 \pm 2.22$ \\
& $\mathrm{X}$ & 32.8 & $1.0 \pm 0.1$ & $124.94 \pm 2.75$ \\
60 & $\mathrm{G}$ & 31.4 & 0 & $87.85 \pm 1.57$ \\
70 & $\mathrm{X}$ & 34.3 & $12.1 \pm 0.9$ & $128.94 \pm 0.43$ \\
& $\mathrm{G}$ & 33.4 & $2.2 \pm 0.4$ & $82.27 \pm 2.31$ \\
80 & $\mathrm{X}$ & 30.4 & $17.9 \pm 0.8$ & $118.55 \pm 4.42$ \\
& $\mathrm{G}$ & 32.7 & $12.9 \pm 0.6$ & $80.45 \pm 1.74$ \\
& $\mathrm{X}$ & 29.9 & $26.3 \pm 1.2$ & $135.21 \pm 2.76$ \\
\hline
\end{tabular}

${ }^{a}$ Represents the type of sugars. $G$ and $X$ represent glucose and xylose, respectively

${ }^{\mathrm{b}}$ Each data represents an average \pm 1 standard deviation of three parallel fermentations

To explore effects of mixed sugar on PPD production, mixed sugars and individual sugars were used for fermentation by Y14. Total concentration of sugar was set to $40 \mathrm{~g} / \mathrm{L}$, and the proportions of xylose used were $0 \%$, $20 \%, 40 \%, 50 \%, 60 \%, 80 \%$, and $100 \%$. The engineered Y14 strain could grow robustly using xylose as the sole carbon source, and PPD yield using xylose was higher than that using mixed sugars or glucose (Table 2). Xylose showed some advantages in PPD production, providing another new fermentation strategy for PPD production by $Y$. lipolytica. Moreover, PPD titer using mixed sugars was greater than that using glucose by S. cerevisiae [35], indicating that xylose was more suitable for PPD production. Because of different cofactor preferences of $\mathrm{XR} / \mathrm{XDH}$, cofactor imbalance may be a limiting factor for PPD production using xylose. In this study, the XR mutation (K270R/N272D) was expressed in Y. lipolytica, which may explain higher PPD production using xylose than that using glucose.

To investigate the reason higher PPD titer were obtained using xylose than using glucose, metabolites, such as acetyl-CoA and squalene, produced by the Y14 strain at $60 \mathrm{~h}$ were analyzed. Acetyl-CoA is an important precursor in the MVA pathway. Cellular acetyl-CoA concentrations may be influenced by carbon sources, contributing to differences in PPD biosynthesis. The concentration of acetyl-CoA produced using xylose was higher than produced using glucose and mixed sugars (Table 2). Furthermore, we analyzed the production of squalene, which is crucial for triterpene biosynthesis via the MVA pathway (Table 2). Higher levels of squalene were produced using xylose as the sole carbon source. In the xylose medium, high acetyl-CoA production resulted in high squalene production compared with that in other media. Thus, PPD titers may be further improved by increasing squalene supply.

\section{Fermentation by the Y14 strain for PPD production in a 5-L bioreactor}

To assess the potential of the Y14 strain as a PPD producer, scale-up experiments were implemented in a 5-L bioreactor using a working volume of 2-L YPD and YPX for cultivating the strain. Xylose and glucose batch fermentations were conducted to test the stability of the engineered strain on fermentation amplification (Fig. 6a, b). Glucose was depleted at $60 \mathrm{~h}$. The highest $\mathrm{OD}_{600}$ was 37.08, and PPD titer was $60.72 \mathrm{mg} / \mathrm{L}$. At $72 \mathrm{~h}, 40 \mathrm{~g} / \mathrm{L}$ xylose was depleted. $\mathrm{OD}_{600}$ was 39.01 , and PPD titer was $98.23 \mathrm{mg} / \mathrm{L}$, which was 1.61-times the titer produced using glucose. PPD titer produced by batch fermentation in the 5-L bioreactor was lower than that produced by shake flask fermentation. This may be because the fermentation conditions were better than that in the shake flasks, resulting in faster substrate consumption and insufficient supply of substrate in the later stages. Yang

Table 2 Biomass and metabolites analysis for Y14 with mixed sugars fermentation

\begin{tabular}{llll}
\hline Xylose/total sugar & OD $_{\mathbf{6 0 0}}$ & PPD titer (mg/L) & Acetyl-CoA (mmol/L) $^{\mathbf{a}}$ \\
\hline 0 & 42.09 & $56.53 \pm 1.23$ & $0.178 \pm 0.012$ \\
$20 \%$ & 41.05 & $68.01 \pm 2.38$ & $0.202 \pm 0.061$ \\
$40 \%$ & 39.45 & $66.12 \pm 1.92$ & $0.197 \pm 0.013$ \\
$50 \%$ & 41.53 & $74.04 \pm 2.17$ & 0.21 \\
$60 \%$ & 41.56 & $72.74 \pm 3.28$ & $0.203 \pm 0.051$ \\
$80 \%$ & 41.5 & $83.23 \pm 4.87$ & $1.973 \pm 0.017$ \\
$100 \%$ & 40.35 & $107.3 \pm 1.93$ & $1.047 \pm 0.082$ \\
\hline
\end{tabular}

a Each data represents an average \pm 1 standard deviation of three parallel fermentations 

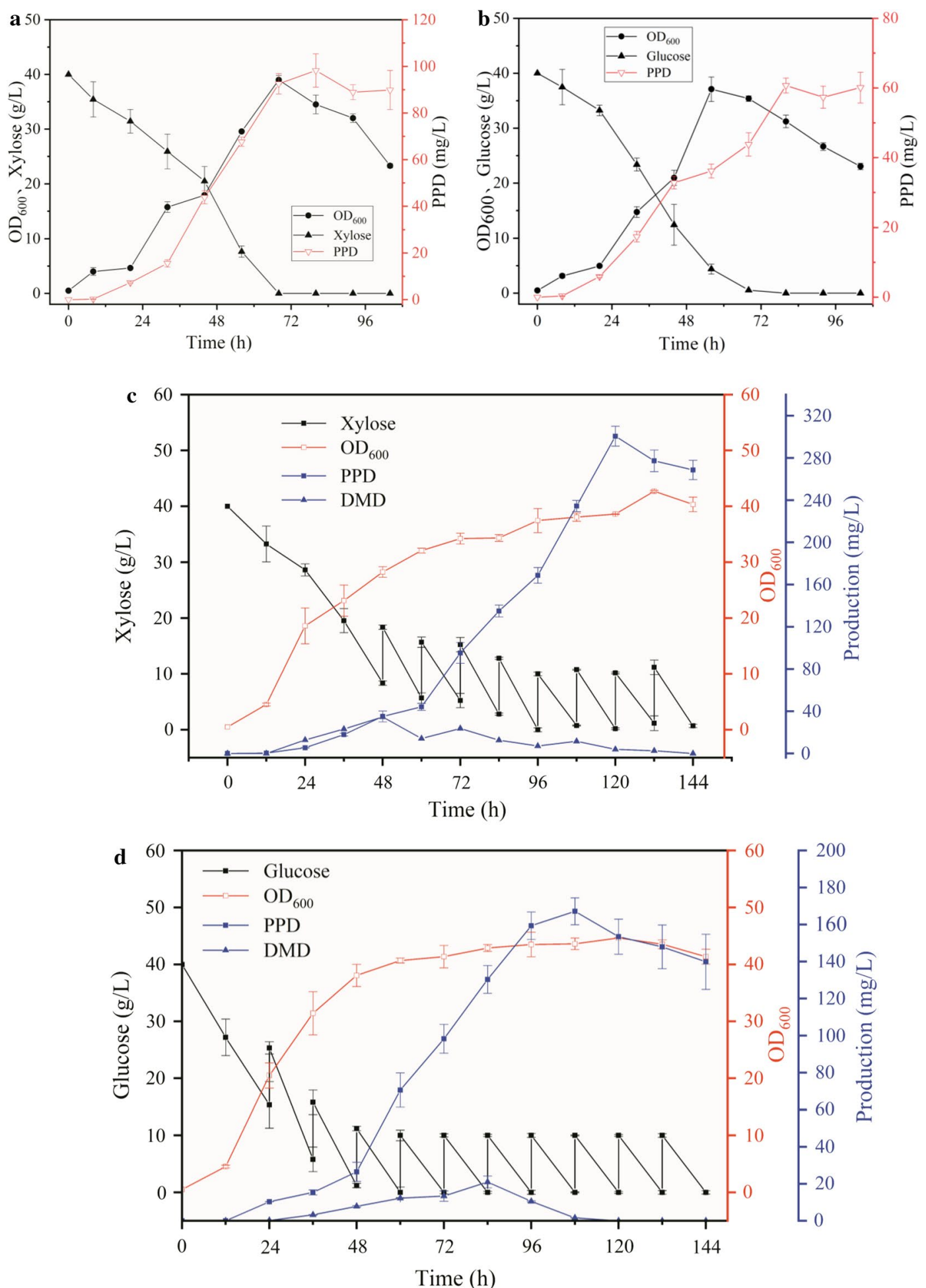

Fig. 6 Fermentation of $Y 14$ for PPD production in a 5-L bioreactor. a Batch fermentation of engineered strain $Y 14$ in a 5-L bioreactor from $x y l o s e . ~ b$ Batch fermentation of engineered strain $\mathrm{Y} 14$ in a 5-L bioreactor from glucose. $\mathbf{c}$ Fed-batch fermentation of engineered strain $\mathrm{Y} 14$ in a 5-L bioreactor from xylose. $\mathbf{d}$ Fed-batch fermentation of engineered strain $\mathrm{Y} 14$ in a 5 -L bioreactor from glucose. Each data represents an average \pm 1 standard deviation of three parallel fermentations 
et al. [26] have constructed an engineered strain of $Y$. lipolytica for $\alpha$-farnesene production and found that the yield in YPD medium by shake flask fermentation could reach $57 \mathrm{mg} / \mathrm{L}$, while that by batch fermentation in $5-\mathrm{L}$ bioreactor could reach $43 \mathrm{mg} / \mathrm{L}$. Sugar consumption was rather fast in the fermenter and could not thus meet the requirements of cell growth and synthesis products. Therefore, for fermentation amplification, we speculated that slower consumption of xylose was more suitable for PPD production than that of glucose.

Fed-batch fermentation in 5-L bioreactor was performed to further increase PPD production (Fig. 6c, d). PPD titer was $300.63 \mathrm{mg} / \mathrm{L}$ [yield, $2.505 \mathrm{mg} / \mathrm{g}$ (sugar); productivity, $2.505 \mathrm{mg} / \mathrm{L} / \mathrm{h}$ ] using xylose. PPD synthesis quickly entered a stable period using glucose, and the highest yield was $167.17 \mathrm{mg} / \mathrm{L}$ [yield, $1.194 \mathrm{mg} / \mathrm{g}$ (sugar); productivity, $1.548 \mathrm{mg} / \mathrm{L} / \mathrm{h}$ ]. Similarly, dammarenediol-II (DMD) production was detected at the same time. DMD remained during xylose fermentation, accumulating at $24 \mathrm{~h}$ and peaking at $84 \mathrm{~h}$, and then gradually decreased. The difference in the fed-batch fermentation results using xylose and glucose may be due to the quick consumption of glucose, which produced amount of acetyl-CoA, but for the limitations of enzymes activity of MVA pathway, carbon sources flowed to other metabolic branches, causing a reduction of acetyl-CoA used for PPD synthesis. With rapid glucose consumption, cells entered a stable phase. Insufficient supply of other precursors, such as acetyl-CoA and NADPH, resulted in slow PPD production. During xylose-feed fermentation, cells continued to grow slowly, and acetyl-CoA or NADPH were continuously supplied for PPD synthesis, reducing the loss of carbon metabolic flux. In addition, DMD accumulated a little, indicating that the supply of DMD was sufficient to be converted to PPD continuously, producing a greater titer. These results indicate that xylose has great potential for PPD production using acetyl-CoA as a precursor. Studies using S. cerevisiae for PPD production using xylose have indicated that xylose fermentation is superior to glucose fermentation in terms of the production of acetyl-CoA. Regulating the metabolic engineering strategy for xylose utilization and product synthesis can enable efficient synthesis of compounds using acetyl-CoA as a precursor and xylose as a substrate.

\section{Conclusion}

This study described a strategy for enhancing PPD production through xylose fermentation with engineered $Y$. lipolytica. Y. lipolytica cannot naturally metabolize xylose; however, introduction of heterologous $\mathrm{XR} / \mathrm{XDH}$ and overexpression of endogenous XKS successfully realized PPD biosynthesis in Y. lipolytica using xylose as the sole carbon source. Biomass and PPD production were further increased by overexpression of tHMG1/ERG9/ ERG20 in MVA pathway and TAL/TKL/TX in xylose metabolic pathway. Regulation of xylose utilization pathway and product synthesis pathways through metabolic engineering can enable efficient synthesis of terpenoid compounds using xylose as a substrate, which can be a strategy for enhancing terpenoid synthesis in Y. lipolytica.

\section{Materials and methods}

Strains, plasmids, and media

Yarrowia lipolytica ATCC 201249 was used as the parental strain for engineering, which was kindly provided by Professor Yingjin Yuan (School of Chemical Engineering and Technology, Tianjin University) [58]. The pINA1269LUL plasmid was used to amplify the marker ura3 with the LoxP sequence, and the pINA1269-CRE plasmid (Additional file 1: Figure S2) carrying a gene encoding CRE protein was used to recycle the marker ura3 (codon-optimized sequences were shown in Additional file 1: Table S1). All Y. lipolytica strains were cultivated in YPD or YPX medium (2\% glucose or $2 \%$ xylose, respectively, $2 \%$ peptone, and $1 \%$ yeast extract) at $30{ }^{\circ} \mathrm{C}$. SC medium $(0.67 \%$ yeast nitrogen base, $2 \%$ glucose, and $2 \%$ agar) lacking leucine or uracil was used for screening $Y$. lipolytica transformants. LB medium ( $1 \%$ tryptone, $0.5 \%$ yeast extract, and $1 \% \mathrm{NaCl}$ ) supplemented with ampicillin $(100 \mathrm{mg} / \mathrm{L})$ was used for culturing Escherichia coli DH $5 \alpha$ containing recombinant plasmids. Feed solution for fermentation contained $400 \mathrm{~g} / \mathrm{L}$ xylose or glucose, $20 \mathrm{~g} / \mathrm{L}$ yeast extract, and $0.5 \mathrm{~g} / \mathrm{L}$ lysine. The components were sterilized separately and combined in a clean environment.

\section{Genetic manipulation}

Codon-optimized xylose reductase XR (XYL1) and $\mathrm{XDH}(X Y L 2)$ derived from S. stipitis were synthesized by Wuhan Genecreate Biological Engineering Co., Ltd. The optimized nucleotide sequences of the genes are presented in Additional file 1: Table S1. The DS, PPDS, and ATR1 genes were synthesized and cloned into pUC57 plasmids by GENEWIZ (Suzhou, China), with codon optimization for $Y$. lipolytica. Endogenous genes encoding XKS $(y l X K S)$, xylose transporter $(T X)$, transketolase $(T K L)$, transaldolase (TAL), truncated 3-hydroxy3-methylglutaryl-coenzyme A reductase (tHMG1), farnesyl pyrophosphate synthase (ERG20), and squalene synthase (ERG9) were amplified using genomic DNA from Y. lipolytica ATCC 201249. Promoters and terminators used in the study were amplified from genomic DNA of Y. lipolytica ATCC 201249 [51]. All primers used to amplify DNA are listed in Additional file 1: Table S2. 


\section{Strain construction}

The expression cassettes used for genome integration were constructed by fusion PCR (Additional file 1: Figure S1). Gene expression cassettes were transformed to $Y$. lipolytica using the LiAc/ssDNA/PEG method, as described previously [52]. The constructed strains are presented in Table 3, and the overall workflow of development of PPD-producing strains was shown Additional file 1: Figure S3.

\section{Adaptation}

Cells were cultured in $5 \mathrm{~mL} \mathrm{SC}$ medium for 7 days. Then, cells were washed using sterile water and transferred to $5 \mathrm{~mL}$ SC medium containing $20 \mathrm{~g} / \mathrm{L}$ xylose (without glucose) for 10 days at $30^{\circ} \mathrm{C}$. Cells were washed again with sterile water and transferred to solid SC medium plates ( $20 \mathrm{~g} / \mathrm{L}$ xylose) to culture single colonies, followed by culture in $5 \mathrm{~mL}$ selection medium containing xylose (without glucose) to select single colonies. The process was repeated $2-3$ times.

\section{Transcriptional gene expression studies by RT-qPCR}

Samples were taken at exponential phase. RNAs was extracted using RNA extraction kit (TIANGEN, China). PrimeScriptTM RT reagent Kit with gDNA Eraser from TaKaRa was used to convent mRNA into cDNA. SYBR ${ }^{\circledR}$ Premix Ex TaqTM II Kit from TaKaRa was used for reverse transcription-quantitative real-time PCR (RT-qPCR). The primers are listed in Additional file 1: Table S3. Triplicate qPCRs were performed. CT (threshold cycle number) values of target genes were normalized using the $\operatorname{erg} 5$ gene as reference. The data obtained were analyzed by applying the $2^{-\Delta \Delta \mathrm{CT}}$ method [59].

\section{Fermentation in shake flasks}

Yarrowia lipolytica strains stored at $-80^{\circ} \mathrm{C}$ in $25 \%$ glycerol were inoculated into $5 \mathrm{~mL}$ YPD medium as the seed culture for $24 \mathrm{~h}$ at $30{ }^{\circ} \mathrm{C}$ while shaking at $220 \mathrm{rpm}$, and the culture was transferred to $250-\mathrm{mL}$ shake flasks loaded with $50 \mathrm{~mL}$ YPD or YPX medium at an initial optical density at $600 \mathrm{~nm}\left(\mathrm{OD}_{600}\right)$ of 0.5 under the same cultivation conditions for 4 days. All shake flask fermentation experiments were performed in three parallel experimental groups.

\section{Batch fermentation}

As seed culture for batch fermentation in 5-L bioreactors (Bailun, Shanghai, China), $5 \mathrm{~mL}$ preculture was transferred to $150 \mathrm{~mL}$ YPD medium in $500-\mathrm{mL}$ shake flasks and cultivated for $24 \mathrm{~h}$ at $30{ }^{\circ} \mathrm{C}$ while shaking at $220 \mathrm{rpm}$. The seed medium was inoculated in a $5-\mathrm{L}$ bioreactor at an initial $\mathrm{OD}_{600}$ of 0.5 in $2 \mathrm{~L} \mathrm{YPD}$ or YPX medium. Temperature was maintained at $30{ }^{\circ} \mathrm{C}$, and $\mathrm{pH}$ was maintained at 6.0 by adding $20 \%$ ammonia water or $3 \mathrm{M} \mathrm{H}_{2} \mathrm{SO}_{4}$. Rotating speed was set at $450 \mathrm{rpm}$, with an air flow rate of $2 \mathrm{vvm}$. For fed-batch fermentation in the 5-L bioreactor, $100 \mathrm{~mL}$ feed solution was added every $12 \mathrm{~h}$ when the sugar was depleted.

\section{PPD extraction and analysis}

PPD was extracted using $n$-butanol, as previously described [35]. The mixture of fermentation broth and $n$-butanol was centrifuged at $11,564 \times g$ for $10 \mathrm{~min}$, and the $n$-butanol phase was collected for analysis. Samples were analyzed by LC/APCI/MS and quantified by HPLC, as previously reported [60]. Standards were purchased from Meilun Biotechnology Co., Ltd (Dalian, China).

\section{Table 3 Strains used in this work}

\begin{tabular}{lll}
\hline Strains & Description & Source \\
\hline Y0 & Yarrowia lipolytica ATCC 201249: MATA, ura3-302, leu2-270, lys8-11, pex17-ha & [59] \\
Y1 & Y0: Ku70 deletion::LUL & This study \\
Y2 & Y1: zeta::exp1p-XYL1-xpr2t, gpd1p-XYL2-lip2t, tef1p-yIXKS-cyc1t & This study \\
Y3 & Y1: zeta: exp1p-yIXYL1-xpr2t, gpd1p-yIXYL2-lip2t, tef1p-yIXKS-cyc1t & This study \\
Y4 & An adaptation of xylose in Y2 & This study \\
Y5 & An adaptation of xylose in Y3 & This study \\
Y6 & The marker ura3 was recycled in Y4 & This study \\
Y7 & Y6: rDNA::tef1p-DS-xpr2t, exp1p-PPDS-mig1t, gpd1p-ATR1-lip2t & This study \\
Y8 & Y6: rDNA::tef1p-DS-xpr2t, exp1p-PPDS-linker-ATR1-lip2t & This study \\
Y9 & The marker ura3 was recycled in Y8. & This study \\
Y10 & Y9: POX1::Fbainp-tHMG1-xpr2t, gpd1p-erg9-cyc1t, exp1p-erg20-lip2t & This study \\
Y11 & The marker ura3 was recycled in Y10. & This study \\
Y12 & Y11:POX2::exp1p-TKL-mig1t, tef1p-TAL-lip2t & This study \\
Y13 & The marker ura3 was recycled in Y12. & This study \\
Y14 & Y13: POX3:: Pfba1-TX-cyc1t & This study \\
\hline
\end{tabular}




\section{Analytical methods}

Cell growth was determined by measuring the $\mathrm{OD}_{600}$ using a UV-VIS spectrophotometer. Concentrations of glucose and xylose were measured using a refractive index detector (Shodex RI-201H) equipped with an Elite P230II pump (Elite Analytical Instruments Co., Ltd., China) and an Aminex HPX-87H column (Bio-Rad, USA). Temperatures of the column and detector were $65{ }^{\circ} \mathrm{C}$ and $40{ }^{\circ} \mathrm{C}$, respectively. The mobile phase was $5 \mathrm{mM} \mathrm{H}_{2} \mathrm{SO}_{4}$ at a flow rate of $0.6 \mathrm{~mL} / \mathrm{min}$. Acetyl-CoA was analyzed using the Acetyl-CoA ELISA Kit (Shanghai Shuangying Co., Ltd., China). Squalene was analyzed using the Elite HPLC system equipped with an Elite P230II high-pressure pump, UV detection at $203 \mathrm{~nm}$, and the Hypersil C18 column $(4.6 \mathrm{~mm} \times 250 \mathrm{~mm}, 5 \mu \mathrm{m})$. Methanol was used as the mobile phase at a flow rate of $1 \mathrm{~mL} / \mathrm{min}$.

\section{Additional file}

Additional file 1. Additional tables and figures.

\section{Acknowledgements}

Not applicable.

\section{Authors' contributions}

YW, XG and DL designed the experiments. YW, SX and ML performed the experiments. YW, XG and WL wrote the manuscript. All authors read and approved the final manuscript.

\section{Funding}

This work was financially supported by the National Natural Science Foundation of China (No. 21878220) and the Major Research Plan of Tianjin [No. 16YFXTSF00460].

\section{Availability of data and materials}

All data generated or analyzed during this study are included in this published article.

Ethics approval and consent to participate

Not applicable.

\section{Consent for publication}

Not applicable.

\section{Competing interests}

The authors declare that they have no competing interests.

\section{Author details}

${ }^{1}$ School of Chemical Engineering and Technology, Tianjin University, Tianjin, People's Republic of China. ${ }^{2}$ Key Laboratory of System Bioengineering (Tianjin University), Ministry of Education, Tianjin, People's Republic of China. ${ }^{3}$ SynBio Research Platform, Collaborative Innovation Center of Chemical Science and Engineering (Tianjin), Tianjin, People's Republic of China.

Received: 20 March 2019 Accepted: 8 May 2019

Published online: 18 May 2019

\section{References}

1. Naik SN, Goud W, Rout PK, Dalai AK. Production of first and second generation biofuels: a comprehensive review. Renew Sustain Energy Rev. 2010;14:578-97.

2. Jeffries TW. Emerging technology for fermenting D-xylose. Trends Biotechnol. 1985;3:208-12.

3. Magasanik B. Catabolite repression. Cold Spring Harb Symp Quant Biol. 1961;26:249.

4. Cirino PC, Chin JW, Ingram LO. Engineering Escherichia coli for xylitol production from glucose-xylose mixtures. Biotechnol Bioeng. 2010;95:1167-76.

5. Lacis LS, Lawford HG. Thermoanaerobacter ethanolicus in a comparison of the growth efficiencies of thermophilic and mesophilic anaerobes. J Bacteriol. 1985:163:1275.

6. Chen T, Liu W, Fu J, Zhang B, Tang Y. Engineering Bacillus subtilis for acetoin production from glucose and xylose mixtures. J Biotechnol. 2013;168:499-505.

7. Wei L, Liu J, Qi H, Wen J. Engineering Scheffersomyces stipitis for fumaric acid production from xylose. Bioresour Technol. 2015;187:246-54.

8. Le Meur S, Zinn M, Egli T, Thny-Meyer L, Ren Q. Production of mediumchain-length polyhydroxyalkanoates by sequential feeding of xylose and octanoic acid in engineered Pseudomonas putida KT2440. BMC Biotechnol. 2012;12:53.

9. Rodriguez GM, Hussain MS, Gambill L, Gao D, Yaguchi A, Blenner M. Engineering xylose utilization in Yarrowia lipolytica by understanding its cryptic xylose pathway. Biotechnol Biofuels. 2016;9:149.

10. Ruiz-Herrera J, Sentandreu R. Different effectors of dimorphism in Yarrowia lipolytica. Arch Microbiol. 2002;178:477-83.

11. Zhao C, Gu D, Nambou K, Wei L, Chen J, Imanaka T, Hua Q. Metabolome analysis and pathway abundance profiling of Yarrowia lipolytica cultivated on different carbon sources. J Biotechnol. 2015:206:42-51.

12. Stephanopoulos G, Tai M, Chakraborty S. Engineered microbes and methods for microbial oil production. 2015.

13. Yeshitila Asteraye T, Chun-Yuan W, Chi-Thanh T, Yi-Hsu J. Lipid production from Yarrowia lipolytica Po1g grown in sugarcane bagasse hydrolysate. Bioresour Technol. 2011;102:9216-22

14. Wei W, Hui W, Markus A, Xiaowen C, Deborah H, Johnson DK, Min Z, Himmel ME. Heterologous expression of xylanase enzymes in lipogenic yeast Yarrowia lipolytica. PLoS ONE. 2014;9:e111443.

15. Blazeck J, Hill A, Liu L, Knight R, Miller J, Pan A, Otoupal P, Alper HS. Harnessing Yarrowia lipolytica lipogenesis to create a platform for lipid and biofuel production. Nat Commun. 2015:5:3131.

16. Ledesma-Amaro R, Lazar Z, Rakicka M, Guo Z, Fouchard F, Crutz-Le Coq AM, Nicaud JM. Metabolic engineering of Yarrowia lipolytica to produce chemicals and fuels from xylose. Metab Eng. 2016;38:115-24.

17. Li H, Alper HS. Enabling xylose utilization in Yarrowia lipolytica for lipid production. Biotechnol J. 2016;11:1230-40.

18. Zhu Z, Zhang S, Liu H, Shen H, Lin X, Fan Y, Zhou YJ, Jin G, Ye M, Zou H. Corrigendum: a multi-omic map of the lipid-producing yeast $R$ hodosporidium toruloides. Nat Commun. 2012;3:1112.

19. Fickers $P$, Benetti $P H$, Waché $Y$, Marty A, Mauersberger S, Smit MS, Nicaud JM. Hydrophobic substrate utilisation by the yeast Yarrowia lipolytica, and its potential applications. FEMS Yeast Res. 2010;5:527-43.

20. Senam S, Barth G. Detection and activation of the Ylt1 retrotransposon in the yeast Yarrowia lipolytica. Berlin: Springer; 2003.

21. Blank LM, Lehmbeck F, Sauer U. Metabolic-flux and network analysis in fourteen hemiascomycetous yeasts. FEMS Yeast Res. 2010;5:545-58.

22. Wasylenko TM, Ahn WS, Stephanopoulos G. The oxidative pentose phosphate pathway is the primary source of NADPH for lipid overproduction from glucose in Yarrowia lipolytica. Metab Eng. 2015;30:27-39.

23. Chen Y, Daviet L, Schalk M, Siewers V, Nielsen J. Establishing a platform cell factory through engineering of yeast acetyl-COA metabolism. Metab Eng. 2013;15:48-54

24. Lian J, Si T, Nair NU, Zhao H. Design and construction of acetyl-CoA overproducing Saccharomyces cerevisiae strains. Metab Eng. 2014;24:139-49.

25. Cao X, Lv YB, Chen J, Imanaka T, Wei LJ, Hua Q. Metabolic engineering of oleaginous yeast Yarrowia lipolytica for limonene overproduction. Biotechnol Biofuels. 2016:9:214.

26. Yang X, Nambou K, Wei L, Hua Q. Heterologous production of a-farnesene in metabolically engineered strains of Yarrowia lipolytica. Bioresour Technol. 2016:216:1040-8. 
27. Li DS, Wu YF, Zhang CB, Sun J, Zhou ZJ, Lu WY. Production of triterpene ginsenoside compound $\mathrm{K}$ in the non-conventional yeast Yarrowia lipolytica. J Agric Food Chem. 2019;67:2581-8.

28. Sun L, Wang Q, Liu X, Brons NHC, Wang N, Steinmetz A, Lv Y, Liao Y, Zheng H. Anti-cancer effects of 20(S)-protopanoxadiol on human acute lymphoblastic leukemia cell lines Reh and RS4;11. Med Oncol. 2011;28:813-21.

29. Popovich DG, Kitts DD. Ginsenosides 20(S)-protopanaxadiol and Rh2 reduce cell proliferation and increase sub-G1 cells in two cultured intestinal cell lines, Int-407 and Caco-2. Can J Physiol Pharmacol. 2004;82:183-90

30. Jian-Li G, Gui-Yuan L, Bai-Cheng H, Bing-Qiang Z, Hongyu Z, Ning W, Chong-Zhi W, Wei D, Chun-Su Y, Tong-Chuan H. Ginseng saponin metabolite 20(S)-protopanaxadiol inhibits tumor growth by targeting multiple cancer signaling pathways. Oncol Rep. 2013;30:292-8.

31. Dai Z, Liu Y, Zhang X, Shi M, Wang B, Wang D, Huang L, Zhang X. Metabolic engineering of Saccharomyces cerevisiae for production of ginsenosides. Metab Eng. 2013;20:146-56.

32. Zhao F, Du Y, Bai P, Liu J, Lu W, Yuan Y. Enhancing Saccharomyces cerevisiae reactive oxygen species and ethanol stress tolerance for high-level production of protopanoxadiol. Bioresour Technol. 2017;227:308-16.

33. Kwak S, Jin YS. Production of fuels and chemicals from xylose by engineered Saccharomyces cerevisiae: a review and perspective. Microb Cell Fact. 2017; 16:82.

34. Kwak S, Kim SR, Xu H, Zhang GC, Lane S, Kim H, Jin YS. Enhanced isoprenoid production from xylose by engineered Saccharomyces cerevisiae. Biotechnol Bioeng. 2017;114:176-85

35. Gao X, Caiyin Q, Zhao F, Wu Y, Lu W. Engineering Saccharomyces cerevisiae for enhanced production of protopanaxadiol with cofermentation of glucose and xylose. J Agric Food Chem. 2018;66:12009-16.

36. Athanasios B, Jean-Marc N, Claude G. An overview of lipid metabolism in yeasts and its impact on biotechnological processes. Appl Microbiol Biotechnol. 2011;90:1193-206.

37. Beopoulos A, Cescut J, Haddouche R, Uribelarrea JL, Molina-Jouve C, Nicaud JM. Yarrowia lipolytica as a model for bio-oil production. Prog Lipid Res. 2009;48:375-87.

38. Guy-Franck R, Alix K, Ingrid L, Bernard D. Comparative genomics of hemiascomycete yeasts: genes involved in DNA replication, repair, and recombination. Mol Biol Evol. 2005;22:1011-23.

39. Lustig AJ. The Kudos of non-homologous end-joining. Nat Genet. 1999;23:130-1.

40. Verbeke J, Beopoulos A, Nicaud JM. Efficient homologous recombination with short length flanking fragments in Ku70 deficient Yarrowia lipolytica strains. Biotechnol Lett. 2013;35:571-6.

41. Schwartz C, Frogue K, Ramesh A, Misa J, Wheeldon I. CRISPRi repression of nonhomologous endjoining for enhanced genome engineering via homologous recombination in Yarrowia lipolytica. Biotechnol Bioeng. 2017;114:2896-906

42. Chum PY, Schmidt G, Saloheimo M, Landowski CP. Transient silencing of dna repair genes improves targeted gene integration in the filamentous fungus Trichoderma reesei. Appl Environ Microb. 2017;83:e00535-17.

43. Watanabe S, Saleh AA, Pack SP, Annaluru N, Kodaki T, Makino K. Ethanol production from xylose by recombinant saccharomyces cerevisiae expressing protein-engineered nadh-preferring xylose reductase from Pichia stipitis. Microbiology. 2007;153:3044-54.

44. Ha SJ, Galazka JM, Rin Kim S, Choi JH, Yang X, Seo JH. Engineered Saccharomyces cerevisiae capable of simultaneous cellobiose and xylose fermentation. Proc Natl Acad Sci. 2011;108:504-9.

45. Liang L, Zhang J, Lin Z. Altering coenzyme specificity of Pichia stipitis xylose reductase by the semi-rational approach casting. Microb Cell Fact. 2007;6:36.

46. Hamacher T, Becker J, Márk G, Bärbel Hahn-Hägerdal B, Boles E. Characterization of the xylose-transporting properties of yeast hexose transporters and their influence on xylose utilization. Microbiology. 2002;148:2783-8.
47. Saloheimo A, Rauta J, Stasyk OV, Sibirny AA, Penttil M, Ruohonen L. Xylose transport studies with xylose-utilizing Saccharomyces cerevisiae strains expressing heterologous and homologous permeases. Appl Microbiol Biotechnol. 2007;74(5):1041-52.

48. Subtil T, Boles E. Competition between pentoses and glucose during uptake and catabolism in recombinant Saccharomyces cerevisiae. Biotechnol Biofuel. 2012;5:14.

49. Liu E, Hu Y. Construction of a xylose-fermenting Saccharomyces cerevisiae strain by combined approaches of genetic engineering, chemical mutagenesis and evolutionary adaptation. Biochem Eng J. 2010;48:204-10.

50. Ryu S, Hipp J, Trinh CT. Activating and elucidating complex sugar metabolism in Yarrowia lipolytica. Appl Environ Microbiol. 2016;82:1334-45.

51. Sun J, Zhang CB, Nan WH, Li DS, Ke D, Lu WY. Glycerol improves heterologous biosynthesis of betulinic acid in engineered Yarrowia lipolytica. Chem Eng Sci. 2018;196:82-96.

52. Guo X, Jie S, Li D, Lu W. Heterologous biosynthesis of (+)-nootkatone in unconventional yeast Yarrowia lipolytica. Biochem Eng J. 2018;137:125-31.

53. Gao S, Tong Y, Zhu L, Ge M, Zhang Y, Chen D, Jiang Y, Yang S. Iterative integration of multiple-copy pathway genes in Yarrowia lipolytica for heterologous $\beta$-carotene production. Metab Eng. 2017;41:192.

54. Ryu S, Trinh CT. Understanding functional roles of native pentose-specific transporters for activating dormant pentose metabolism in Yarrowia lipolytica. Appl Environ Microbiol. 2018;84:e02146-17.

55. Walfridsson M. Xylose-metabolizing Saccharomyces cerevisiae strains overexpressing the TKL1 and TAL1 genes encoding the pentose phosphate pathway enzymes transketolase and transaldolase. Appl Environ Microbiol. 1995;61:4184-90.

56. Abdel-Rahman MA, Tashiro Y, Zendo T, Sonomoto K. Effective (+)-lactic acid production by co-fermentation of mixed sugars. J Biotechnol. 2010;150:347-8.

57. Stephan L, Shuyan Z, Na W, Christopher R, Yong-Su J. Development and physiological characterization of cellobiose-consuming Yarrowia lipolytica. Biotechnol Bioeng. 2015;112:1012-22.

58. Papanikolaou S, Muniglia L, Chevalot I, Aggelis G, Marc I. Yarrowia lipolytica as a potential producer of citric acid from raw glycerol. J Appl Microbiol. 2010;92:737-44.

59. Xue C, Zhang X, Yu Z, Zhao F, Wang M, Lu W. Up-regulated spinosad pathway coupling with the increased concentration of acetyl-CoA and malonyl-CoA contributed to the increase of spinosad in the presence of exogenous fatty acid. Biochem Eng J. 2013;81:47-53.

60. Zhao F, Bai P, Liu T, Li D, Zhang X, Lu W, Yuan Y. Optimization of a cytochrome $\mathrm{P} 450$ oxidation system for enhancing protopanaxadiol production in Saccharomyces cerevisiae. Biotechnol Bioeng. 2016;113:1787-95.

\section{Publisher's Note}

Springer Nature remains neutral with regard to jurisdictional claims in published maps and institutional affiliations.

Ready to submit your research? Choose BMC and benefit from:

- fast, convenient online submission

- thorough peer review by experienced researchers in your field

- rapid publication on acceptance

- support for research data, including large and complex data types

- gold Open Access which fosters wider collaboration and increased citations

- maximum visibility for your research: over $100 \mathrm{M}$ website views per year

At BMC, research is always in progress.

Learn more biomedcentral.com/submissions 